\title{
Nonpolar solvation dynamics for a nonpolar solute in room temperature ionic liquid: a nonequilibrium molecular dynamics simulation study
}

\author{
SANDIPA INDRA ${ }^{\mathrm{a}}$ and SNEHASIS DASCHAKRABORTY ${ }^{\mathrm{b}, *}$ (1) \\ ${ }^{a}$ 19, Nischintapur Lane, Santipur, Nadia, West Bengal 741 404, India \\ ${ }^{b}$ Department of Chemistry, Indian Institute of Technology Patna, Patna, Bihar 801 103, India \\ E-mail: snehasis@iitp.ac.in
}

MS received 11 September 2017; revised 4 November 2017; accepted 5 November 2017; published online 30 January 2018

\begin{abstract}
Nonpolar solvation dynamics of a nonpolar diatomic solute in a room temperature ionic liquid (RTIL) has been followed via nonequilibrium molecular dynamics (MD) simulation. Frank-Condon type excitation of the solute, previously in equilibrium in RTIL solvent, has been modelled by abruptly changing the Lennard-Jones (LJ) diameter of the solute atoms and thereby disrupting the equilibrium situation. The rearrangement of the RTIL solvent molecules, which has been seen to be mostly contributed by the solute's first solvation shell, around the excited solute results overall spectral narrowing and biphasic decay of the solvation energy; a dominant and very rapid process having sub-100 fs relaxation time, followed by a slower one relaxing at a timescale of $\sim 5 \mathrm{ps}$. A mode-coupling theory based calculation is also used to obtain the nonpolar solvation relaxation function for a model nonpolar solute dissolved in model RTIL solvent. The theoretical relaxation decay is not in very good agreement with the simulated nonequilibrium solvation response function; the theory predicts the short time relaxation component slower and the longtime component faster than those of the simulated nonequilibrium relaxation. We have also checked the validity of the linear response theory (LRT) for nonpolar solvation in RTIL by looking at the equilibrium solvation energy correlation in the RTIL solvent in presence of the ground state (GS) and the excited state (ES) solute. Apparent breakdown of the LRT in the present case elucidates the probable disagreement between the theoretical and simulated nonequilibrium nonpolar solvation response functions.
\end{abstract}

Keywords. Solvation dynamics; nonpolar solvation; ionic liquid; molecular dynamics; linear response theory.

\section{Introduction}

Room temperature ionic liquids (RTILs) are interesting new materials with multiple fascinating properties. An increasing number of studies are putting forward newer applications of these RTILs; as the reaction medium, ${ }^{1,2}$ in energy applications, ${ }^{3}$ as $\mathrm{CO}_{2}$ capturing material, ${ }^{4,5}$ etc. These applications are directly connected to the key macroscopic properties of RTILs, which too originate from the structural and dynamical properties at the molecular level. This is the rationale of growing interest of understanding molecular structure and dynamics of RTILs using experiment, theory and computer simulation.

\footnotetext{
*For correspondence

The solvation dynamics of RTILs for an electronically excited solute molecule-which is generally fluorescent-have been studied over the years using the Stokes shift dynamics method ${ }^{6-22}$ in order to detect the solvation behavior, the knowledge of which is the key to judge a particular RTIL as a potential reaction medium. Due to the fact that the dipole moment of the electronically excited state of the solute generally increases substantially (e.g., the dipole moment of C153 probe increases by $\sim 7 \mathrm{D}$ upon electronic excitation by a particular wavelength radiation), ${ }^{23}$ the solute primarily probes the solute-RTIL dipole-dipole and ion-dipole interactions guided solvation of RTILs. The dipolar solvation response function calculated by following the time-dependent shift of the fluorescence emission 
spectrum of the excited probe molecule for imidazolium and pyrrolidinium ILs shows biphasic decay: the faster component ( $\sim 20 \%$ of the total response) relaxes exponentially with sub-picosecond time constant ( $\sim 100-700 \mathrm{fs})$, and the slower component relaxes stretched-exponentially (stretched exponent $\ll 1$ ) with time constant ranging over a few picoseconds to several nanoseconds. ${ }^{10-23}$ While it is somewhat settled that the origin of the slower relaxation is usually due to the slower rotation of the dipolar cations and/or the translation of the ions, the origin of the ultrafast time constant is still debated. ${ }^{24-42}$ Multiple modes of molecular movements can be responsible for this ultrafast relaxation, including the hydrogen-bond (H-bond) libration, ${ }^{25-31}$ faster rotation of the dipolar cation, ${ }^{24-29}$ fast translation of small constitute anion, ${ }^{10,37-42}$ etc. Recent classical density functional based theoretical calculations ${ }^{24-31}$ supported the experimental findings and thereby put forward possible origins of different solvation relaxation timescales.

More recent three-pulse photon echo peak shift (3PEPS) measurements using oxazine-4 probe in several RTILs have reported unexpectedly fast solvation. ${ }^{43,44}$ The measured relaxation time scales are way faster than those obtained in the time-resolved fluorescence Stokes shift measurements (TRFSS). ${ }^{10-13}$ It's highly unlikely that the traditional dynamical modes, discussed in the preceding paragraph, can completely explain the origin of this ultrafast solvation. This anomaly reminds the experimental report of ultrafast solvation using transient hole-burning technique for a wide variety of viscous solvents. ${ }^{45-47}$ It was observed that the solvation of dimethyl-s-tetrazine-a nonpolar solute-is governed by an unprecedented fast relaxation mechanism. The relaxation was sometimes more than two orders of magnitude faster than the traditional diffusive re-orientational relaxation of polar solvation near dipolar solutes. The key origin for this very fast relaxation was later understood to be nonpolar interaction between the excited solute and the solvent and therefore the associated solvation was termed as nonpolar solvation. ${ }^{45-53}$ This opens up the possibility of identifying nonpolar solvation as the key relaxation mechanism found in the recent 3PEPS measurement for RTILs. Furthermore, the reported Stokes shift value is $\sim 200-500 \mathrm{~cm}^{-1}, 43,44$ which is much smaller than that found in TRFSS measurement using C153. ${ }^{10-13}$ This is probably due to the low polarity of both the ground and excited state oxazine-4 probe molecule. This motivated one of us to investigate the problem using mode coupling based theory on non-polar solvation dynamics of different RTILs at a model nonpolar solute. ${ }^{30}$ The theoretical relaxation timescales were in semi-quantitative agreement with the measured ones and thus the theoretical study drew an important conclusion that the 3PEPS and TRFSS experiments are probably reconnoitring different components of the total solvation energy relaxation of a laser-excited dye in a given RTIL.

Although the above mode-coupling theory based calculation was somewhat successful in understanding the nonpolar solvation relaxation of the oxazine- 4 probe in RTILs, the theory is not absolutely free from problems, particularly when one uses it for a complex solvent like RTILs. The key approximations in this theory are as follows: (i) the solvent molecules are perfectly spherical in shape, (ii) the molecules are uniformly distributed in space, and (iii) the LRT holds perfectly so that the non-equilibrium solvation response function measured experimentally can be written as equilibrium solvation energy correlation function. The first approximation is valid when the theory is used for smaller molecular liquids and therefore the shape can be well approximated as spherical. But the known complexities of RTIL molecules somewhat question the spherical approximation. This approximation for RTIL molecules is more of a concern, particularly for nonpolar solvation. This is due to the fact that the nonpolar solvation is dominated by the nonpolar interaction-e.g., LJ interaction, which is short-ranged and thereby primarily contributed by the nearest neighbour solvent IL molecules. Interestingly, the similar spherical approximation of RTIL molecule is proven to be valid for dipolar solvation as the theoretically predicted solvation response semi-quantitatively agrees with measured one. ${ }^{24,25,30}$ This can be understood from the fact that dipolar solvation, unlike the nonpolar one, takes into account largely the longer ranged soluteIL dipole-dipole interaction and therefore average out the local shape factor significantly. Similarly, the second approximation is also questionable due to the fact that the RTIL is not a microscopically homogeneous at all and possesses spatial and temporal heterogeneities. ${ }^{32,54}$ The importance of local interaction in nonpolar solvation makes the approximation even more questionable. The approximation, that the LRT holds correctly, has generally proven to be valid for solvation of a dipolar solute in common dipolar solvents and RTILs. ${ }^{39,55}$ But the theory severely breaks down for nonpolar solvation response function in common dipolar solvents when the solute is subjected to sudden perturbation in size parameters, as done in computer simulation for obtaining nonequilibrium solvation response. ${ }^{56}$ These limitations of the theory motivate us to study the nonpolar solvation dynamics for explicit all-atom model of RTIL molecule near an excited nonpolar solute using nonequilibrium molecular dynamics simulation technique and address 
the possible contrast between the mode coupling theory and all-atom molecular dynamics simulation.

In this paper, we determine by nonequilibrium simulation the nonpolar solvation energy relaxation of a diatomic solute in $[\mathrm{Bmim}]\left[\mathrm{PF}_{6}\right]$ —an imidazolium ILby suddenly changing the LJ parameters of the solute, the way to mimic the Frank-Condon type excitation. Then the rearrangement of IL molecules around the excited solute and thereby the solvation energy relaxation has been monitored over time by looking at the time-dependent Stokes shift value. We have also compared the mode coupling based theoretical solvation energy relaxation with the simulated one in order to check the rationality of the theory in this RTIL. The virtue of using all-atom model of IL in the present simulation has given us the opportunity of looking at the individual contributions of different segments of the IL to the total Stokes shift and thereby understanding their relative significance in the whole process. We have also calculated the equilibrium solvation energy correlation function from equilibrium trajectory for both GS and ES of the solute in order to compare with the nonequilibrium solvation response function and thus check the validity of LRT for nonpolar solvation in RTIL.

\section{Model description and simulation details}

In this section, we first provide the simulation details and the equilibration of the system. The section ends with a discussion on how the trajectories are generated for analyses.

\subsection{Simulation details and system equilibration}

The simulated system consists of 128 pairs of [Bmim $^{+}$cation (see Scheme 1a) and $\left[\mathrm{PF}_{6}\right]^{-}$anion (Scheme 1b) as the solvent and one nonpolar solute, modelled as a simple rigid diatomic molecule (Scheme 1c). Traditionally, this type of solute model has been used for decades in MD simulation for probing solvation behavior in dipolar liquid. ${ }^{55-57}$ In addition, the solute model having rotational degrees of freedom is more realistic than a single atomic solute model and takes into account the solute's rotational contribution towards the total solvation dynamics.

Initial gas-phase structures of the $[\mathrm{Bmim}]^{+}$cation and $\left[\mathrm{PF}_{6}\right]^{-}$anion are obtained by optimizing at B3LYP/6 $-311+$ $\mathrm{G}^{* *}$ level of theory and basis set with the GAMESS package. ${ }^{58} 128$ pairs of the optimized ions and the diatomic solute molecule, the structure of which is created by the bond distance parameter between the two atoms, are then placed in a cubic box; the configuration is generated using PACKMOL package. ${ }^{59}$ The required box length is calculated from the previously simulated equilibrium density of this RTIL (4.705 mol dm${ }^{-3}$ ) for the same force field and the temperature $(298 \mathrm{~K})$ we use here. ${ }^{60}$
For the RTIL solvent, we use the force field developed by Canongia-Lopes et al., based on the OPLS-AA framework. ${ }^{60}$ Detailed description of the potential form including the meaning of the terms is presented elsewhere. ${ }^{61}$ The rationale of using this force field, apart from the fact that the use of this force field results into much less computational cost compared to that of the polarizable model, is its proven predictability of the measured solvation and dielectric relaxation behavior of a dipolar solute dissolved in RTILs. ${ }^{33,34,38,39} \mathrm{An}$ alternate model reducing the charges of the RTIL ions to enhance the mobility also does exist. This model is based on the understanding that due to the induced polarization between the ions of RTIL the columbic charges of the ions are less than whole numbers $( \pm 1)$. However, a study on applicability of various models of RTIL in two different dynamical limits, local and collective, shows that the reduced charge model is not at all a good choice when the local interaction is important, for example in single molecule rotational motion of the dipole. ${ }^{62}$ Nonpolar solvation being dominated by the interaction between the solute and the nearest neighbor RTIL solvent molecules is predominantly a local phenomenon. This justifies the use of this simpler yet useful model for the RTIL ions. The solute is modelled as a rigid molecule having two identical LJ atoms of diameter $(\sigma) 3.61 \AA$ and LJ well depth $(\varepsilon) 1.41 \mathrm{KJ} / \mathrm{mol}$. These values represent the GS of the solute. Therefore, the solute and the RTIL ions interact only via LJ interaction: $V_{12}^{L J}=4 \varepsilon_{12}\left[\left(\sigma_{12} / r_{12}\right)^{12}-\left(\sigma_{12} / r_{12}\right)^{6}\right]$; the cross terms $-\sigma_{12}$ and $\varepsilon_{12}$-are calculated using LorentzBerthelot rule. We use GROMACS package ${ }^{63}$ for simulating the entire system in the box. Periodic boundary condition has been employed on all sides of the box. We optimize the entire system using steepest descent algorithm for 1000 steps before $10 \mathrm{~ns}$ equilibrium simulation run, of which the first $5 \mathrm{~ns}$ simulation is run using NPT ensemble and the next 5 ns simulation is continued with NVT ensemble. This $10 \mathrm{~ns}$ simulation is carried out in order to get the proper equilibrated structure. In both cases, we use Verlet leap-frog algorithm for integrating Newton's equations of motion with $1 \mathrm{fs}$ time step. While Nose-Hoover thermostat ${ }^{64,65}$ with coupling constant 0.5 ps is used for controlling the temperature at $298 \mathrm{~K}$ and Berendsen barostat ${ }^{66}$, with the same coupling constant of $0.5 \mathrm{ps}$ is used for controlling the pressure at 1 bar. The long-ranged electrostatic interactions are handled using Particle Mesh Ewald summation technique. ${ }^{67}$

The equilibrated system is then continued to run for another $1 \mathrm{~ns}$ under the same conditions, discussed above before we finally start running multiple nonequilibrium trajectories, which will be discussed now.

\subsection{Simulation trajectory details}

During the $1 \mathrm{~ns}$ equilibrium trajectory with the GS solute, we save configurations every after $10 \mathrm{fs}$, the time which is required to clearly resolve the presumably very fast nonpolar solvation dynamics (see Sect 3.2). Using this trajectory we calculate inter-atomic radial distribution functions $(g(r))$ of the RTIL solvent molecules, presented in Section S1 of 

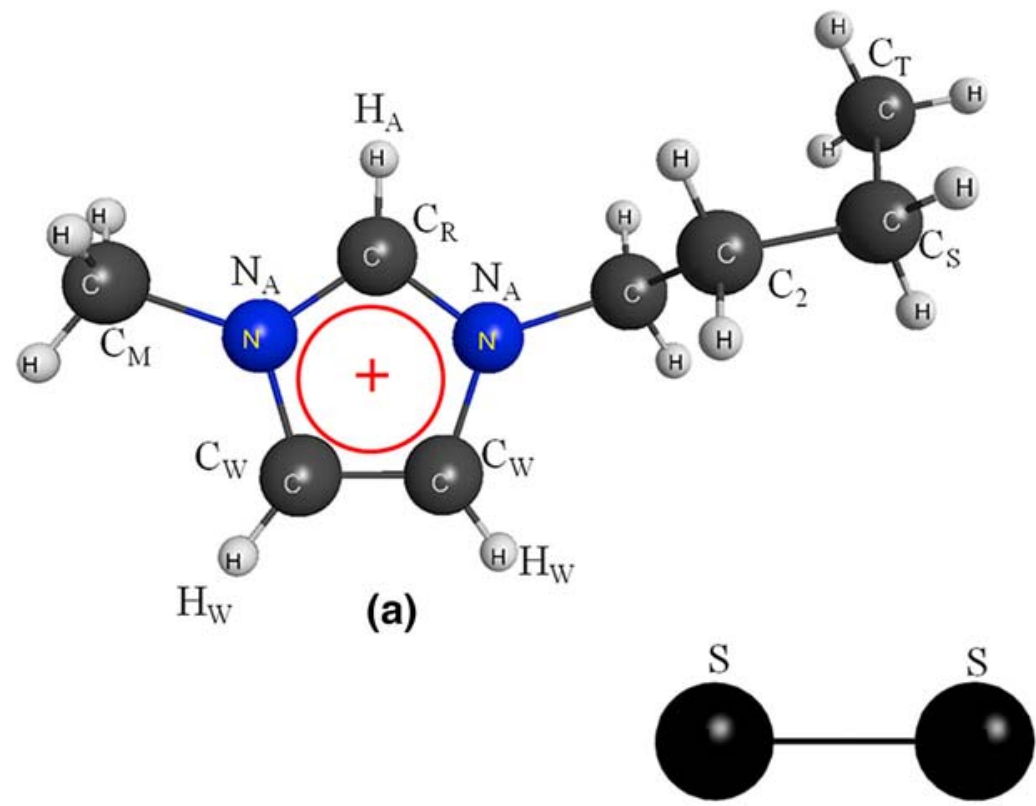

(c)

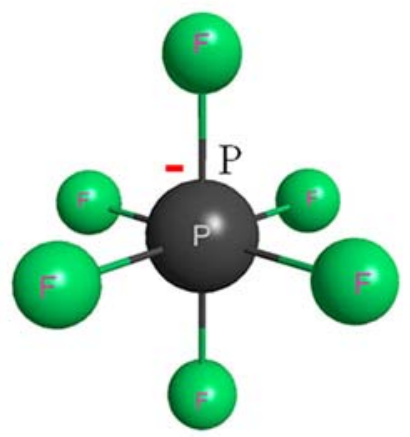

(b)

Scheme 1. The all-atom model of the RTIL, $[\mathrm{Bmim}]^{+}\left[\mathrm{PF}_{6}\right]^{-}$and the diatomic solute.

Supplementary Information (SI). The simulated $g(r)$ s are in excellent agreement with those presented in earlier studies, indicating proper equilibration of the system. ${ }^{68-70}$ This trajectory is also used to get the equilibrium solvent configuration around the GS solute, which also assists to make a reference for the configuration after the nonequilibrium situation is created by suddenly changing the LJ parameter(s) of the solute.

100 nonequilibrium trajectories are initiated at a regular interval of $30 \mathrm{ps}$ from a long equilibrium trajectory of duration $3.0 \mathrm{~ns}$ starting from the end point of the previous $1 \mathrm{~ns}$ equilibrium trajectory. The first nonequilibrium trajectory is thus initiated from the final configuration of the previous $1 \mathrm{~ns}$ equilibrium trajectory. All the nonequilibrium trajectories are started by increasing LJ diameters of the solute atoms by $20 \%$ of its GS value, keeping LJ well depth $(\varepsilon)$ unchanged. The new LJ diameter for the solute is thus $(\sigma)$ $4.33 \AA$. We call the solute with the new LJ parameters as ES solute. The 100 different nonequilibrium trajectories serve two purposes. First, they provide a sampling of different initial solvent environments, a characteristic decisive for a set of such nonequilibrium trajectories. Also, they gradually increase the length of the equilibration process, which is desired for a slower solvent like [Bmim][PF 6$]$ RTIL. The total duration of the equilibrium trajectory in presence of the GS solute is thus equal to $4 \mathrm{~ns}$, which is used for the equilibrium solvation analysis. We extend the equilibrium trajectory to $20 \mathrm{~ns}$ for obtaining reasonably smooth equilibrium structure of RTIL molecules around the GS solute. We calculate the time-dependent quantities in Section 3 by averaging over all these 100 nonequilibrium trajectories at a common reference time at $t=0$, the starting point of the trajectories. The lengths of all the trajectories are $30 \mathrm{ps}$, during which temperature constraining is lifted so that the natural dynamics are not affected anyway. We will see in Section 3 that the 30 ps duration of the nonequilibrium trajectories is sufficient for completing the nonpolar solvation around the ES solute. The configurations have been saved in the interval of $5 \mathrm{fs}$, which is essential for getting fine details of the time-dependent results, especially for ultrafast nonpolar solvation. By convergence study (Section S2 of SI) we have shown that the set of 100 nonequilibrium trajectories is enough for present sampling purpose.

One of the nonequilibrium trajectories is then extended for another $20 \mathrm{~ns}$ in order to obtain the equilibrium trajectory including new RTIL solvent configurations around the solute in its ES. Configurations from both the equilibrium trajectories in presence of GS and ES solute have been saved every after 10 fs. The equilibrium solvation picture around the GS and the ES solute are extracted from the equilibrium trajectories of $20 \mathrm{~ns}$ each.

\section{Results and Discussion}

In this section, we first compare the equilibrium solvation structure of the solute between its GS and the ES. The equilibrium average peak shift is shown next. The time-dependent analysis is started by looking at the time-dependent spectral width function and peak shift. We then focus on the relative contributions from the various RTIL sites and the solute's solvation shells towards the total time-dependent peak shift. Simulated nonequilibrium nonpolar solvation response function is then presented and compared with the solvation energy-energy correlation function, obtained from 
mode-coupling theory based calculation. The section ends with a discussion on examining the validity of LRT for nonpolar solvation of the solute in the RTIL solvent.

\subsection{Structural change}

Figure 1 presents $g(r)$ s between the solute and the center of mass of the RTIL cation, $[\mathrm{Bmim}]^{+}$and the anion, $\left[\mathrm{PF}_{6}\right]^{-}$both at the GS and the ES of the solute. Clearly, the $g(r)$ peaks shift towards longer $r$ values for ES, which is simply due to the increase of the $\sigma$ value of the solute atoms. Also, the RTIL ions are more structurally organized around the ES solute than that around the GS one. This stems from the fact that the increase of $\sigma$ value of the solute atoms increases the attractive part of the potential and thereby increases the structural order of the RTIL solvent molecules around the solute. The change of the solvation structure around the solute molecule is associated with the solvation dynamics, the primary objective of the study. A closer comparison between Figure $1 \mathrm{a}$ and $1 \mathrm{~b}$ reveals that the change of $g(r)$ peak height is more pronounced for solute- $\left[\mathrm{PF}_{6}\right]^{-}$than that for solute-[Bmim $]^{+}$.
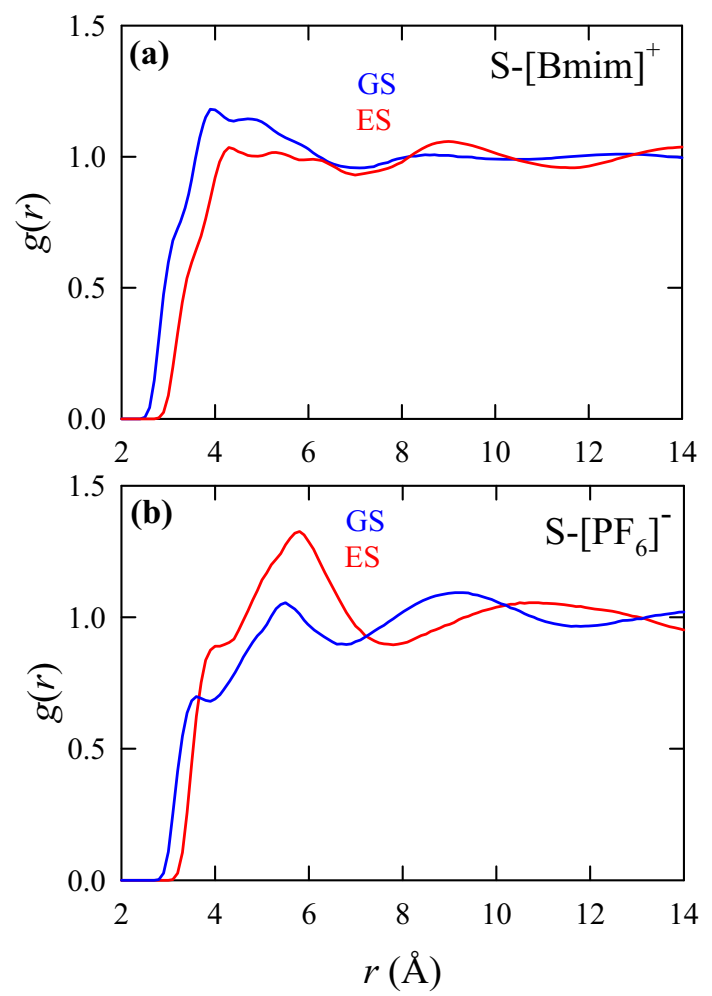

Figure 1. Comparison between the solute-solvent $g(r)$ at the GS and the ES of the solute: $g(r)$ between (a) COM of the solute and COM of [Bmim $^{+}$cation and (b) COM of the solute and $\mathrm{COM}$ of $\left[\mathrm{PF}_{6}\right]^{-}$anion of the RTIL (See Scheme 1 for the atom labelling).
In order to get further insight about the equilibrium solvation structure of the solute in $[\mathrm{Bmim}]\left[\mathrm{PF}_{6}\right]$, we have shown in Figure 2 the $g(r)$ between the solute atoms and some specific atoms of the RTIL. $g(r) \mathrm{s}$ between the solute and the remaining alkyl carbon atoms of the alkyl chain of $[\mathrm{Bmim}]^{+}$cation are presented in Figure S3 of SI. Visibly the solvation structure around the solute changes appreciably upon the excitation of the solute. Generally, the first peaks shift towards longer interatomic distance and the overall solvation structures look more ordered ones. This reiterates the direct proportionality between the increase of $\sigma$ value of the solute atoms with the increase of both repulsive and attractive part of the potential. Comparative analysis of the solvent's site-specific $g(r)$ shows that at GS the solute stays more in the hydrophobic domain (near $\mathrm{C}_{T}$ ), as evidenced by the most intense $g(r)$ peak for the solute-RTIL. This is in accordance with the fact that the solute is nonpolar and therefore prefers the nonpolar domain of the RTIL, which is the hydrophobic side chain. Comparison of the intensities of the $g(r)$ peaks between the solute and the alkyl side chains of the $[\mathrm{Bmim}]^{+}$cation (Figure $2 \mathrm{c}$ and Figure S3 of SI) shows that the GS solute stays closer to $\mathrm{CT}$ than $\mathrm{C} 2$ or $\mathrm{CS}$; the order of the peak intensities is $\mathrm{CT}>\mathrm{CS}>\mathrm{C} 2$. Interestingly, similar behavior is observed for solvation structure of longer chain RTILs $\left(\left[\mathrm{C}_{10} \mathrm{mim}\right][\mathrm{Cl}]\right.$ and $\left.\left[\mathrm{C}_{10} \mathrm{mim}\right]\left[\mathrm{PF}_{6}\right]\right)$ around ${ }^{129} \mathrm{Xe}$ solute probe. $^{71}$

The solvent environment around the excited solute changes appreciably. The sharp decrease of the first $g(r)$ peak height between the ES solute and RTIL $\mathrm{C}_{T}$ indicates that the ES solute, unlike the GS solute, is not overwhelmingly situated near the hydrophobic domain. Also, the first peak heights between the ES solute and the cationic head group atoms $\left(\mathrm{N}_{A}, \mathrm{C}_{R}\right)$ and $\left[\mathrm{PF}_{6}\right]^{-}$anion are more intense than those with GS solute. Therefore, the solvent environment is somewhat diverse around the ES solute as indicated by the similar first peak positions and heights of different $g(r) \mathrm{s}$ in Figure 2. The diverse solvent environment around the ES solute corroborates with the features, one generally expects from a solvent like RTIL, which possesses great extent of spatiotemporal microscopic heterogeneity. ${ }^{72-77}$

We now focus on the average dynamic red shift $\Delta E(t)$ (see Section S4 of SI for the definition), the value of which is calculated using the equation, $\langle\Delta E(t)\rangle=$ $\left\langle V_{E S, I L}(t)\right\rangle-\left\langle V_{G S, I L}(t)\right\rangle$, where $V_{G S, I L}^{L J}$ and $V_{E S, I L}^{L J}$ are the LJ interactions between the GS and ES solute respectively with the RTIL solvent. The normalized probability distributions of $\Delta E$ values in the presence of GS and ES solute are presented in Figure 3. These two distributions are calculated from the two equilibrium trajectories 

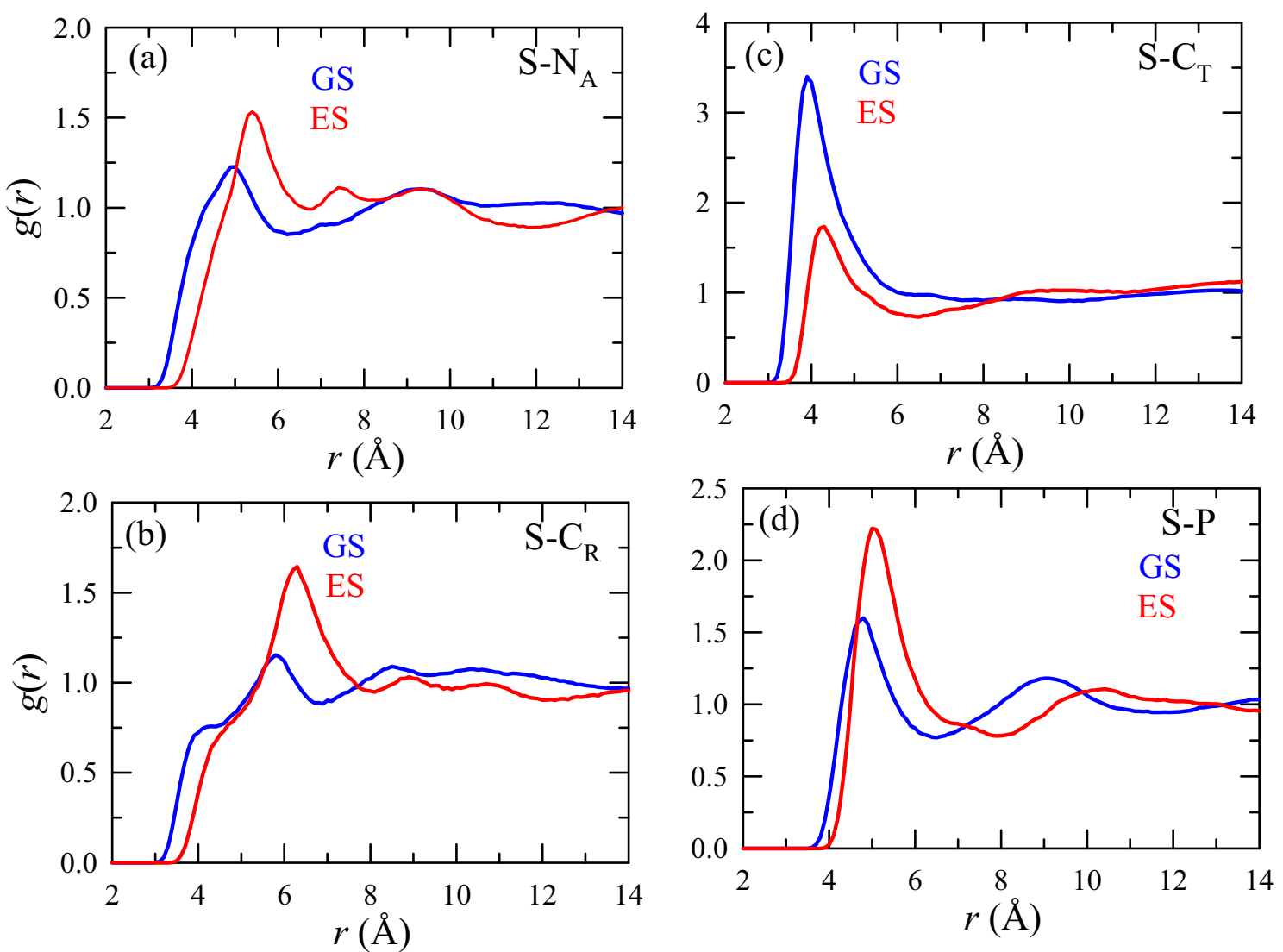

Figure 2. $g(r)$ between solute atoms and the various atoms of the RTIL ions. The $g(r)$ for GS solute is labelled as blue and that for the ES solute is labelled as red. See Scheme 1 for the atom-labelling.

(4 ns), one involving the GS solute and the other involving the ES solute. Clearly, neither of the distributions are Gaussian in nature indicating the presence of local structural heterogeneity around the solute. It's worth mentioning that the same distributions for the dipolar solvation of a diatomic solute in a model polar solvent were seen to be Gaussian. ${ }^{55}$ The GS distribution is set to be centered at $\Delta E=0$ and therefore the peak position of the ES distribution measures the equilibrium averaged shift. The ES distribution peak is shifted towards the red end by $\sim 3000 \mathrm{~cm}^{-1}$, indicating the average red shift. Note we have obtained much larger Stokes shift compared to the reported shift in 3PEPS measurement. ${ }^{43,44}$ This is clearly due to the high increase of the $\sigma$ values by $20 \%$ of its initial value. Despite the fact that smaller increase of $\sigma$ value might result into the smaller shift, we increased the value of $\sigma$ of the solute so high. The rationale is following. Figure 3 clearly demonstrates that the distribution of shift in the presence of GS solute is far wide (the width at half maximum is $\sim 4000 \mathrm{~cm}^{-1}$ ). This distribution in presence of the ES solute should be amply different from that of the GS solute in order to get a clear time-dependent shift (see Section 3.2) with minimum error especially when

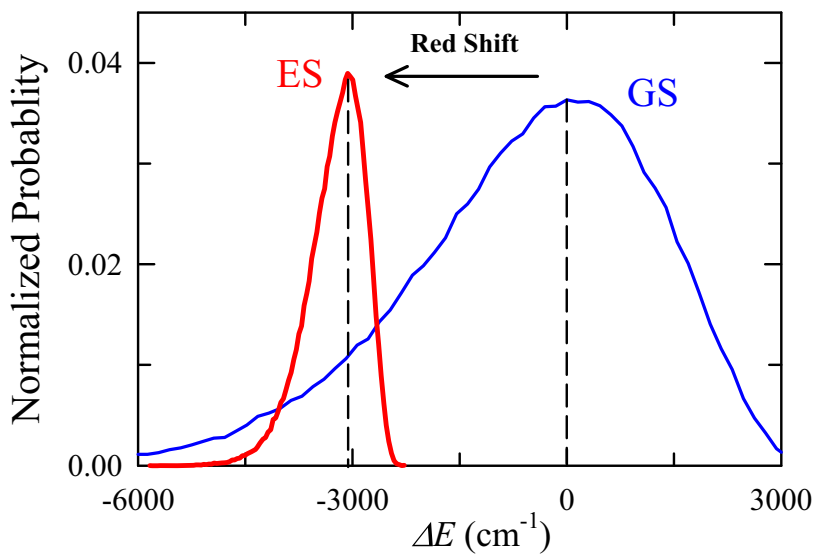

Figure 3. Normalized equilibrium probability distributions of $\Delta E$ values in the presence of the GS solute (blue line) and the ES solute (red line). The sampling is done after every $10 \mathrm{fs}$ over $4 \mathrm{~ns}$ equilibrium trajectories. The vertical dashed lines indicate the maxima of the peaks.

we have limited number of nonequilibrium trajectories. Choice of smaller increment of $\sigma$ value of the solute would result into significant overlapping between the two distributions in Figure 3 for GS and ES solute and 
thereby the time-dependent shift would have low signal/noise ratio. However, the solvation energy relaxation time scales, the primary objective of this paper, should not have much effect on this extent of change of the $\sigma$ value.

Further inspection of Figure 3 also shows that the initial $\Delta E$ probability distribution appropriate for the GS solute is much wider than the final $\Delta E$ probability distribution appropriate for the ES solute. This suggests TRFSS spectrum should show an overall timedependent narrowing, which we will examine in the next subsection. This spectral narrowing must be directly related to the larger solvent force constant near the minima of the solvent-free energy curve for the ES solute. The larger solvent force constant for the ES solute is again due to the increased interaction as indicated by the more ordered solvation structure of the ES solute than that of the GS solute.

\subsection{Nonpolar solvation dynamics}

We now examine the time-dependent width function followed by simulated nonequilibrium nonpolar solvation dynamics. We then discuss another well-established approach namely, the mode-coupling theory based calculation to obtain the nonpolar solvation response function and finally compare the theoretical relaxation time scales with the simulated ones in order to understand the origin of the simulated relaxation timescales.

The time-dependent width function, $W(t)$, is calculated by looking at the standard deviation of the $\Delta E$ sample for 100 different non-equilibrium trajectories; $W(t)=\left\langle(\Delta E)^{2}\right\rangle-\langle\Delta E\rangle^{2}$. Figure 4 presents timedependent $W(t)$, which shows very fast decay as it almost reaches equilibrium in 150 fs. This ultrafast decay must be seen also in time-dependent Stokes shift, as we will see now.

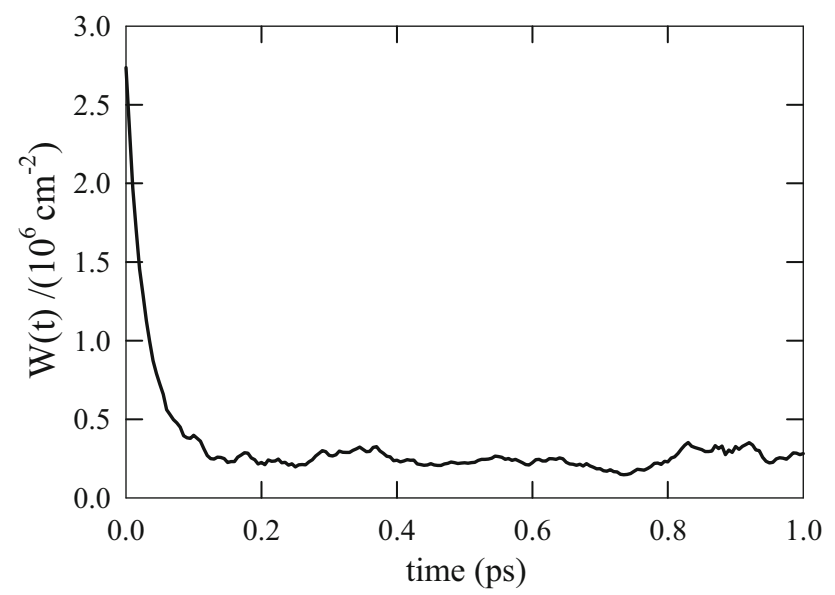

Figure 4. The width function $W(t)$ as a function of time.
The time-dependent shift $\langle\Delta E(t)\rangle$ (see Eq. 4 in Section $\mathrm{S} 4$ of $\mathrm{SI}$ ) has been plotted in Figure $5 \mathrm{a}$ as a function of time. The overall dynamic Stokes shift is $\sim 3580 \mathrm{~cm}^{-1}$ (obtained by time averaging at long time decay), which is slightly higher than the equilibrium average red shift $\left(3000 \mathrm{~cm}^{-1}\right)$. The probable reason for this difference is lesser sampling in the time-resolved study than that in average equilibrium ones calculated in Figure 3. Note that while sampling of only 100 different configurations is considered for nonequilibrium calculation, normalized equilibrium probability distributions of $\Delta E$ values in the presence of the GS and ES solute are calculated using $\sim 5 \times 10^{5}$ configurations (once in every 10 fs during 5 ns equilibrium trajectory). Furthermore, the very broad distribution of $\Delta E$ in presence of the GS solute (width at the half maximum $\sim 4000 \mathrm{~cm}^{-1}$ ) introduces uncertainty in $\Delta E(t=0)$ no matter what. All these factors cause the observed difference between the equilibrium and time-dependent shift values. Note that the large dynamic Stokes shift is obtained as in the case of equilibrium Stokes shift, detailed in Section 3.1, where we have also discussed the possible reason for obtaining this large Stokes shift.

Now, we focus on the relative contributions of the total dynamic Stokes shift from different solvation shells and different segments of the RTIL molecule in order to gain further knowledge about the origin of this dynamic Stokes shift value. In Figure 5b, we have plotted the two time-dependent shifts: (i) that due to the interaction between the solute and the solvent RTIL molecules which are only in the $1^{\text {st }}$ solvation shell of the solute and (ii) the one due to the interaction between the solute and the solvent RTIL molecules which are outside the $1^{\text {st }}$ solvation shell. The separation between the solvation shells is entirely based on the distance criteria (the solute - the center of mass of RTIL molecule) which was reflected in Figure 1. For example, the first solvation shell of the solute is considered up to the distance where the first minimum appears after the first peak of $g(r)$.

Figure $5 \mathrm{~b}$ very clearly shows that the total dynamic Stokes shift $\sim 3580 \mathrm{~cm}^{-1}$ is remarkably contributed by the first solvation shell of the solvent RTIL; the value is $\sim 3534 \mathrm{~cm}^{-1}$ (obtained by time averaging at long time decay), which is almost $\sim 99 \%$ of the total shift. The rest of the shift $\left(\sim 46 \mathrm{~cm}^{-1}\right)$ is contributed by the solvation shells beyond the first one. This is consistent with the fact that the LJ interaction-the only solute-solvent interaction in the system-is short ranged and decays very rapidly as a function of internuclear separation $\left(\propto 1 / r^{-6}\right)$.

We now investigate the relative contributions of four key segments of the RTIL - the methyl group (Me), the 

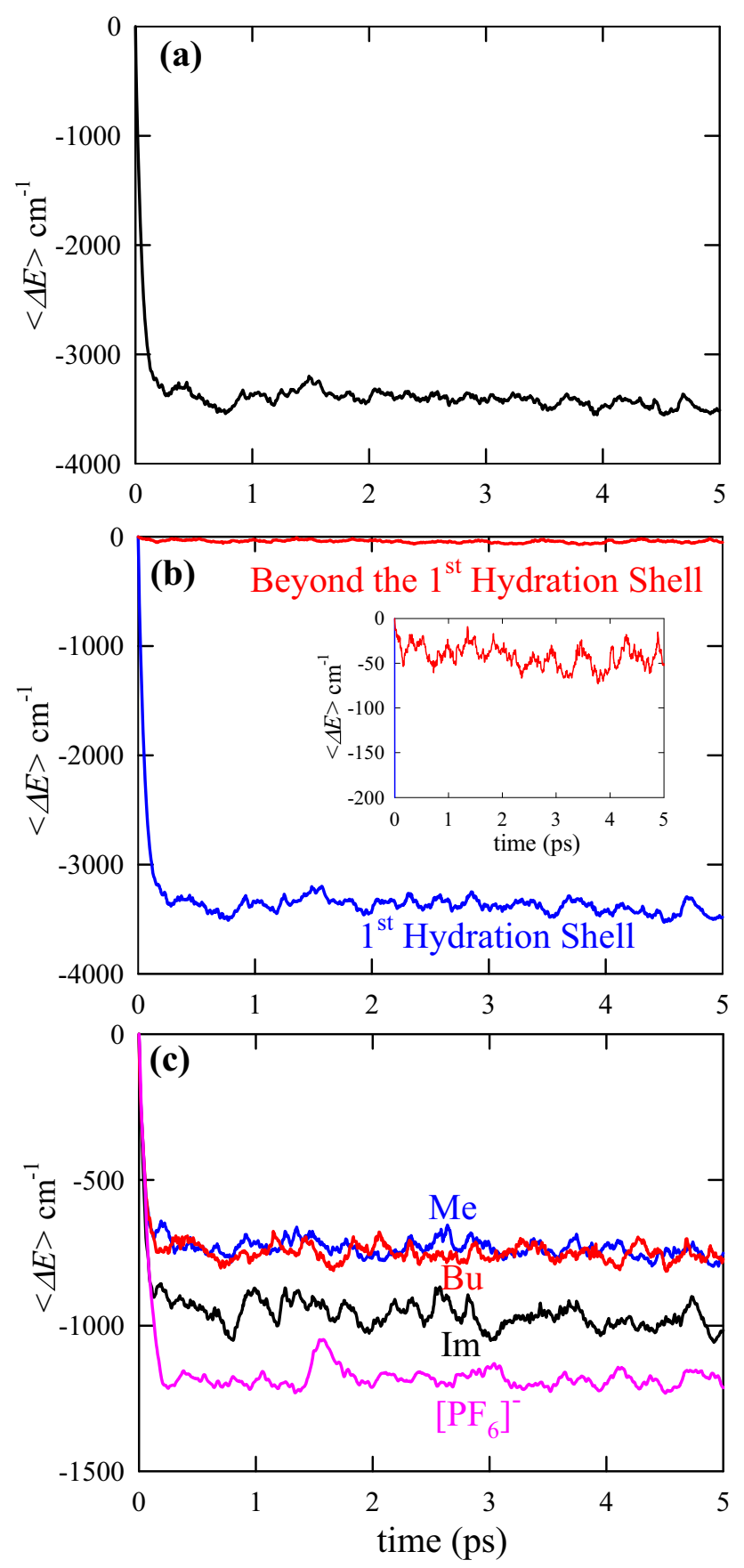

Figure 5. (a) Trajectory-averaged time-dependent total Stokes shift $\langle\Delta E(t)\rangle$ as a function of time. (b) Separate contributions of the shift from $1^{\text {st }}$ solvation shell (blue line) and from the outer shells (red line). The inset shows the latter contribution more clearly. (c) Division of the total shift in contributions from separate segments of $[\mathrm{Bmim}]\left[\mathrm{PF}_{6}\right]$ RTIL molecule. The blue, red, black and pink lines represent the contributions from the methyl group, the butyl group, the imidazolium ring, and the $\left[\mathrm{PF}_{6}\right]^{-}$anion.

imidazolium ring $(\mathrm{Im})$, the butyl group $(\mathrm{Bu})$, and the $\left[\mathrm{PF}_{6}\right]^{-}$anion-towards the total shift. This particular segmentation is done on the basis of an established foursite model of RTIL, proposed by Roy and Maroncelli. ${ }^{78}$

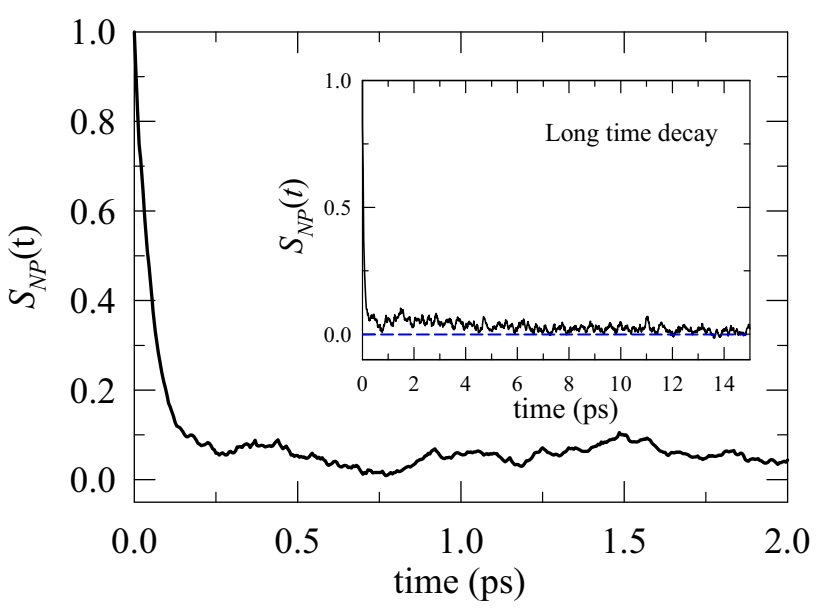

Figure 6. Nonequilibrium nonpolar solvation response decay, $S_{N P}^{N e q m}(t)$, of the nonpolar solute in $\left[\mathrm{Bmim}^{\mathrm{N}}\right]\left[\mathrm{PF}_{6}\right]$ RTIL. The longtime decay of $S_{N P}^{N e q m}(t)$ is shown in the inset. The blue horizontal dashed line indicates the value " 0 " for $S_{N P}^{N e q m}(t)$.

Interestingly, the $\left[\mathrm{PF}_{6}\right]^{-}$anion has the highest contribution of all. The order of increasing contribution from the segments towards the total shift is as follows: $\left[\mathrm{PF}_{6}\right]^{-}>$ $\mathrm{Im}>\mathrm{Bu} \approx \mathrm{Me}$

Figure 5a clearly shows that the shift is very much rapid as it is almost over by $\sim 150$ fs. However, a very subtle shift occurs at longer times up to $\sim 15 \mathrm{ps}$, which we examine now. The time-dependent nonequilibrium solvation response function $S_{N P}^{N e q m}(t)$ has been displayed in Figure 6. This has been calculated using the following equation:

$S_{N P}^{N e q m}(t)=\frac{\langle\Delta E(t)\rangle-\langle\Delta E(\infty)\rangle}{\langle\Delta E(0)\rangle-\langle\Delta E(\infty)\rangle}$,

where " $<>$ " indicates the quantity averaged over various initial conditions. Note that the $\Delta E(t)$ values during the last $5 \mathrm{ps}$ of the nonequilibrium trajectories are used for obtaining the $\langle\Delta E(\infty)\rangle$ values. The very fast dynamics involved in this relaxation process is again quite apparent. In addition, there is a slower component as well, relaxing up to $\sim 15 \mathrm{ps}$. The slower part of the relaxation is clearly visible in the inset of Figure 6. In order to elucidate further, we have fitted the relaxation function with a biexponential equation of the form, $a_{1} \exp \left(-t / \tau_{1}\right)+a_{2} \exp \left(-t / \tau_{2}\right)$, where $a_{1}+a_{2}=1$. While the faster component having the contribution of $\sim 94 \%$ of the total shift relaxes at a timescale of $\sim 50 \mathrm{fs}$, the slower component with only $\sim 6 \%$ contribution has the relaxation time constant of $\sim 5.07$ ps. Note that 3PEPS measurement using oxazine-4 solute probe molecule, which has a very small change of dipole moment upon photo-excitation, reported the biphasic 
solvation relaxation with time constants of $\sim 150 \mathrm{fs}$ and $\sim 2.20 \mathrm{ps}$, which are in the same order of magnitudes with the simulated time scales. ${ }^{43,44}$ This indicates that the measured solvation can instigate largely from nonpolar solvation due to a sudden change of nonpolar interaction between the solute and the RTIL solvent, which can be caused by the Frank-Condon type excitation of the oxazine- 4 solute molecule. Note that 3PEPS measurements are associated with the line-broadening function, determined by the total solvation energy correlation function, ${ }^{43}$ both polar and nonpolar components of solute-solvent interactions contribute to the total peak shift.

Equilibrium nonpolar solvation energy correlation function can also be obtained using mode-coupling based semi-molecular level theory. ${ }^{30,48,49,79}$ Recently, this theory has been used for this system in order to get an insight on the origin of the relaxation timescales, found in the 3PEPS experiment. ${ }^{43,44}$ We now discuss the theory briefly and compare with the present simulated nonequilibrium solvation response function.

The nonequilibrium solvation response function within the realm of LRT can be equated to the normalized nonpolar solvation energy correlation function as,

$S_{N P}^{\text {Theory }}(t)=\frac{C_{N P}(t)}{C_{N P}(t=0)}$,

where $C_{N P}(t)$ - the un-normalized nonpolar solvation energy-energy time correlation function-can be expressed as the sum of a short time binary part and a long time collective part assuming complete decoupling between them. ${ }^{30,48,49}$ Therefore, one can write:

$C_{N P}(t)=C_{N P}^{B}(t=0) \exp \left[-\left(t / \tau_{B}\right)^{2}\right]+C_{N P}^{\rho \rho}(t)$.

The first and the second term of the right-hand side of Eq. 3 are due to the interactions at binary (solute-IL) and collective limit. The faster binary component relaxes at a timescale of $\tau_{E}^{B}$, which can be written as ${ }^{30,48,49}$

$\tau_{B}^{=} \sqrt{\frac{-2 C_{N P}^{B}(t=0)}{\ddot{C}_{N P}^{B}(t=0)}}$,

where $\ddot{C}_{N P}^{B}(t=0)=\frac{d^{2} C_{N P}^{B}(t=0)}{d t^{2}}$. The binary part is basically determined by the solute-IL solvent LJ interaction potential $\left(v_{12}(r)\right)$, where the solvent distribution around the solute can be approximated by the solute-IL solvent pair correlation function, $g_{12}(r)$, weighted by the solvent density $(\rho)$. Therefore, $C_{N P}^{B}(t=0)$ and $\ddot{C}_{N P}^{B}(t=0)$ can be calculated using the following equations respectively: ${ }^{30,48,49}$

$C_{N P}^{B}(t=0)=4 \pi \rho \int_{0}^{\infty} d r r^{2}\left[v_{12}(r)\right]^{2} g_{12}(r)$, and

$$
\begin{aligned}
& \ddot{C}_{N P}^{B}(t=0) \\
& \quad=\frac{4 \pi \rho}{m^{*}} \int_{0}^{\infty} d r r^{2}\left[v_{12}(r)\right]^{2} \nabla_{r} g_{12}(r) \nabla_{r} v_{12}(r) .
\end{aligned}
$$

Where $m^{*}$ denotes the reduced mass of the solutesolvent composite system.

The slower part which has the contribution from the collective density fluctuation can be expressed as, ${ }^{30,48,49}$

$C_{N P}^{\rho \rho}(t)=\frac{\left(k_{B} T\right)^{2} \rho}{6 \pi^{2}} \int_{0}^{\infty} d k k^{2} c_{12}^{2}(k) S(k, t)$,

where $c_{12}(k)$ is wave-number, $k$ dependent two particles direct correlation function and $S(k, t)$ is the dynamic solvent structure factor of RTIL solvent. Note that the details of the calculation of $g_{12}(r), c_{12}(k)$, and $S(k, t)$ in Eq. 5-7 for this system are discussed elsewhere. ${ }^{30}$

We now use the above theoretical formulation to calculate the NP solvation response function $S_{N P}^{\text {theory }}(t)$ for a nondipolar solute dissolved in $[\mathrm{Bmim}]\left[\mathrm{PF}_{6}\right]$ IL assuming the size of the solute molecule same as the RTIL solvent. The parameters necessary for the calculation like the sizes of the ions, the density of the RTIL, the dielectric constant of the solvent RTIL, etc., are available in the previous paper. ${ }^{30} S_{N P}^{\text {Theory }}(t)$ is plotted as a function of time in Figure 7. However, for comparison, we also include the simulated nonequilibrium solvation response function $S_{N P}^{N e q m}(t)$, already shown in Figure 6. Visibly, the initial part of $S_{N P}^{\text {Theory }}(t)$ decays slower than that of $S_{N P}^{N e q m}(t)$ but at the later time, during the slower relaxation, both decay reasonably at a similar rate. Fitting with a function, sum of Gaussian and exponential functions having the following form:

$S(t)=a_{1} \exp \left(-\left(t / \tau_{1}\right)^{2}\right)+a_{2} \exp \left(-t / \tau_{2}\right)$

we get the timescale-associated with the initial Gaussian relaxation- $\left(\tau_{1}\right) \sim 225$ fs with a contribution $\sim 89 \%$ and the final slower exponential part having only $\sim 11 \%$ contribution relaxing at a timescale of $\sim 2.76 \mathrm{ps}$. Clearly, the faster component is $\sim 5$ times slower and the slower component is $\sim 2$ times faster than the simulated relaxation. However, the theoretical and simulated amplitudes of the two modes of the relaxation are in close agreement with each other. The disagreement between the timescales probably stems from multiple reasons. Firstly, the calculation-being based on modecoupling theory - has considered the RTIL molecule as a spherical particle, a very simplified model for the RTIL molecule, which has a complex structure altogether. The local complexity in the solute-RTIL solvent interaction is therefore completely ignored. This may lead to serious problem in predicting particularly the initial 


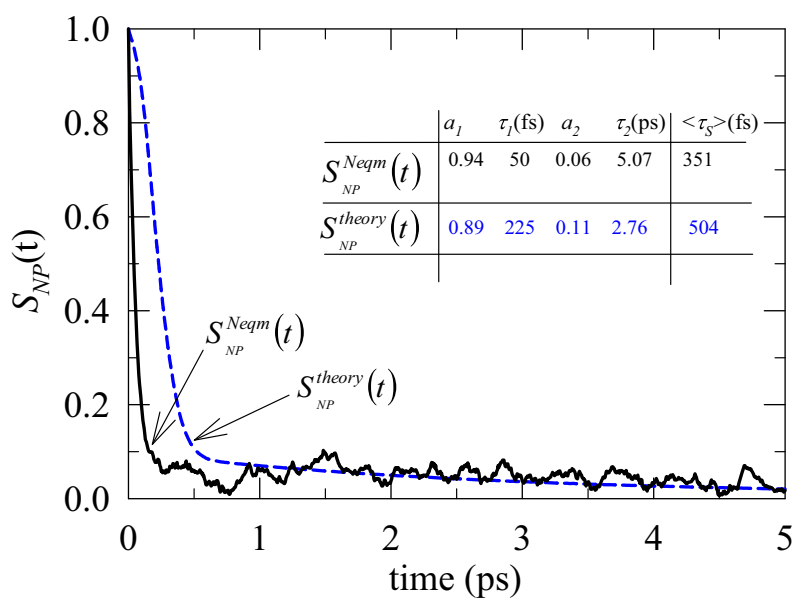

Figure 7. Time-dependent nonequilibrium nonpolar solvation response function, $S_{N P}^{N e q m}(t)$, of the nonpolar solute in [Bmim] $\left[\mathrm{PF}_{6}\right]$ RTIL (black solid line) and the equilibrium solvation response function from the theoretical calculation (blue dashed line) using Eq. 7. The fitting parameters of $S_{N P}^{N e q m}(t)$ and of $S_{N P}^{\text {Theory }}(t)$ are listed in the table (inset). Note that while the $S_{N P}^{\text {Theory }}(t)$ is fitted with a function, sum of Gaussian and exponential functions (see Eq. 8), $S_{N P}^{N e q m}(t)$ is fitted with a simple biexponential function.

response, which is due to the binary interaction between the solute and the RTIL solvent. Secondly, the theory is based on the approximation that the RTIL is microscopically homogeneous. This approximation may lead to serious error in estimating the dynamic structure factor $(S(k, t))$ of the RTIL, the key factor in regulating the collective density fluctuation of the solvent. Therefore, the slower component of $S_{N P}^{\text {Theory }}(t)$ can deviate from the simulated one simply due to an inaccuracy of the calculated $S(k, t)$. Note that the present simulationbeing based on the well-established all-atom model of $[\mathrm{Bmim}]\left[\mathrm{PF}_{6}\right]$ - takes into account the detailed structural and dynamical behavior of the RTIL. Finally, by assuming the validity of LRT for nonpolar solvation in RTIL the theory considers the solvation energy-energy correlation to be same as the nonequilibrium solvation response. Therefore, the invalidity of the LRT may lead to inaccuracy in calculated solvation response function and the associated relaxation timescales. In the next subsection, we discuss this in more details.

\subsection{Validity of LRT}

In the study of solvation dynamics of an ion pair in a polar solvent by Carter and Hynes it was shown that two forms of LRT can be employed to make the connection between the nonequilibrium solvation response function and equilibrium solvation energy correlation function. ${ }^{55}$ These are equilibrium solvation energy correlation for neutral pair and ion pair. Along the same line we can also connect the nonequilibrium solvation response function with the equilibrium solvation energy correlation function either for the GS $\left(S_{N P}^{E q m, G S}(t)\right)$ or for the ES solute $\left(S_{N P}^{E q m, E S}(t)\right)$.

The equilibrium solvation energy correlation functions for the GS and the ES solute are calculated using the following equations: ${ }^{55}$

$S_{N P}^{E q m, G S}(t)=\frac{\langle\delta \Delta E(0) \delta \Delta E(t)\rangle_{G S}}{\left\langle(\delta \Delta E(0))^{2}\right\rangle_{G S}}$,

and

$S_{N P}^{E q m, E S}(t)=\frac{\langle\delta \Delta E(0) \delta \Delta E(t)\rangle_{E S}}{\left\langle(\delta \Delta E(0))^{2}\right\rangle_{E S}}$,

where $\delta \Delta E(t)=\Delta E(t)-\langle\Delta E(t)\rangle$, the fluctuation of the time-dependent shift from time-averaged value, and " $<>$ " denotes the equilibrium average obtained from equilibrium simulation trajectories in presence of GS and ES solute.

Therefore the validity of LRT for NP solvation in $[\mathrm{Bmim}]\left[\mathrm{PF}_{6}\right]$ RTIL implies that

$$
\begin{aligned}
S_{N P}^{N e q m}(t) & =S_{N P}^{E q m, G S}(t)=\frac{\langle\delta \Delta E(0) \delta \Delta E(t)\rangle_{G S}}{\left\langle(\delta \Delta E(0))^{2}\right\rangle_{G S}} \\
& =S_{N P}^{E q m, E S}(t)=\frac{\langle\delta \Delta E(0) \delta \Delta E(t)\rangle_{E S}}{\left\langle(\delta \Delta E(0))^{2}\right\rangle_{E S}} .
\end{aligned}
$$

Figure 8 displays the three response functions, $S_{N P}^{\text {Neqm }}(t)$, $S_{N P}^{E q m, G S}(t)$, and $S_{N P}^{E q m, E S}(t)$ as a function of time. Although, two equilibrium correlations $-S_{N P}^{E q m, G S}(t)$ and $S_{N P}^{E q m, E S}(t)$-decay almost similarly with similar timescales and amplitudes, $S_{N P}^{\text {Neqm }}(t)$ - most importantly-does not match either with $S_{N P}^{E q m, G S}(t)$ or $S_{N P}^{E q m, E S}(t)$. Apparently, the equilibrium correlations decay slower than the nonequilibrium correlation. In order to view this with more clarity, we fit the two equilibrium relaxation decays with biexponential functions of the form, $a_{1} \exp \left(-t / \tau_{1}\right)+a_{2} \exp \left(-t / \tau_{2}\right)$, where $a_{1}+a_{2}=1$. The fitting parameters, including the average solvation time $\left(\left\langle\tau_{S}\right\rangle\right)$ are described in Table 1 . Although the relaxation time constants of the equilibrium correlations are not very different from those of $S_{N P}^{N e q m}(t)$, the amplitude of the slower part of the relaxation of the formers is almost double than that of the latter. This makes the overall equilibrium relaxations much slower than that of $S_{N P}^{N e q m}(t)$. Clearly, this indicates a breakdown of LRT for nonpolar solvation of a nonpolar solute in this RTIL. 


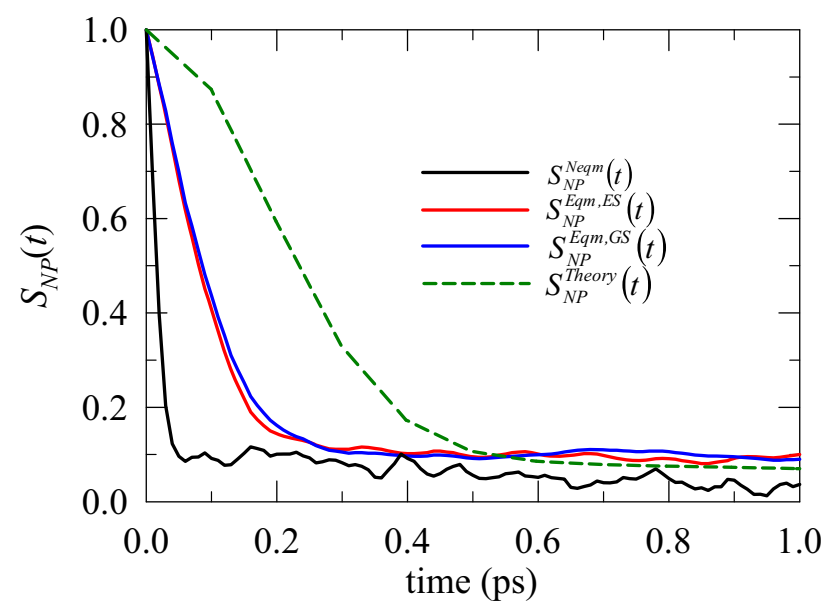

Figure 8. Time-dependent NP solvation response functions from various routes. The representation is as follows: the simulated nonequilibrium solvation response $S_{N P}^{N e q m}(t)$ (black solid line), the equilibrium solvation energy correlation functions for the GS $\left(S_{N P}^{E q m, G S}(t)\right.$ (blue solid line) and for the ES solute $\left(S_{N P}^{E q m, E S}(t)\right)$ (red solid line) and the theoretical solvation response function, $S_{N P}^{\text {Theory }}(t)$ (green dashed line).

Table 1. The fitting parameters-including the average solvation time $\left(\left\langle\tau_{S}\right\rangle\right)$-for the four solvation response functions, shown in Figure 8. The solvation response timescales, measured by 3PEPS experiment, are also listed.

\begin{tabular}{|c|c|c|c|c|c|}
\hline$S_{N P}(t)$ & $a_{1}$ & $\tau_{1}(\mathrm{fs})$ & $a_{2}$ & $\tau_{2}(\mathrm{ps})$ & $\left\langle\tau_{\text {solv }}\right\rangle(\mathrm{fs})$ \\
\hline$S_{N P}^{N e q m}(t)$ & 0.94 & 50 & 0.06 & 5.07 & 351 \\
\hline$S_{N P}^{E q m, G S}(t)$ & 0.90 & 93 & 0.10 & 6.33 & 716 \\
\hline$S_{N P}^{E q m, E S}(t)$ & 0.90 & 89 & 0.10 & 6.18 & 698 \\
\hline$S_{N P}^{T \text { heory }}(t)$ & 0.89 & 225 & 0.11 & 2.76 & 504 \\
\hline$S^{\text {Expt }}(t)$ & & 150 & & 2.26 & \\
\hline
\end{tabular}

In order to elucidate further we have also included in Figure 8 the theoretical solvation response function $S_{N P}^{\text {Theory }}(t)$ and in Table 1 the corresponding fitting parameters. Note that $S_{N P}^{\text {Theory }}(t)$ is fitted with a function - sum of Gaussian and exponential functions-having the form of Eq. 8. Apparent from Figure 8 and Table 1 is that the theory predicts the initial part of the solvation slowest and the later part of the solvation fastest of all. Interestingly, the amplitudes of the two relaxation components of $S_{N P}^{\text {Theory }}(t)$ match very well with the equilibrium relaxations, indicating the better predictability of the theory for the simulated equilibrium solvation relaxation. The overall relaxation of, $S_{N P}^{\text {Theory }}(t)$, decays at a rate in between the equilibrium and nonequilibrium solvent relaxation.
Lastly, the theoretical and simulated timescales are in semi-quantitative agreement with the experimentally measured timescales. This corroborates with the understanding that the origin of the ultrafast timescales, observed in 3PEPS measurement in RTILs, might be the nonpolar interaction between excited solute and RTIL molecules.

\section{Conclusions}

We have presented here results for nonequilibrium nonpolar solvation dynamics of an ES solute dissolved in $[\mathrm{Bmim}]\left[\mathrm{PF}_{6}\right]$ RTIL. The Frank-Condon type excitation of the solute due to its sudden change of the LJ diameter creates a perturbation in the equilibrium solvation of the GS solute in the RTIL. The solvent molecules - located primarily in the first solvation shell-therefore needs to rearrange until a new equilibrium is attained for the solvation of the ES solute. This causes an overall time-dependent spectral narrowing and shifts towards smaller frequency. The process is biphasic with the initial dominant component relaxing at a timescale of $\sim 50$ fs followed by a very weak slower decay having a timescale of $\sim 5$ ps. These timescales are not very different from the ones observed in a 3PEPS measurement using Oxazine-4 solute as the probe molecule. ${ }^{43,44}$ This indicates a possibility that the 3PEPS measurement probes predominantly nonpolar part of the solvation. We have also used a simple mode-coupling based approach to calculate solvation energy-energy correlation function and then compared with the simulated nonequilibrium relaxation in order to verify the theoretical prediction. ${ }^{30,48,49}$ Breakdown of the LRT has been viewed for nonpolar solvation in RTIL while comparing the simulated nonequilibrium solvent response function with equilibrium response functions, calculated using equilibrium trajectories in presence of GS and ES solute.

Note that in this study we have excited the solute by changing only $\sigma$ of the solute atoms without affecting their $\varepsilon$ parameter. This is due to the fact that the $\mathrm{LJ}$ potential-being a function of the $12^{\text {th }}$ and $6^{\text {th }}$ order of $\sigma$ respectively for repulsive and attractive part of the potential-is much more sensitive towards the change of $\sigma$ value compared to that with the change of $\varepsilon$ parameter, which linearly changes the LJ interaction.

It's worth mentioning that temperature dependent studies for the solvation dynamics can be very important in the sense that the viscosity-a function of temperature-can in principally affect the nonpolar solvation dynamics in general and the slower part of the decay to be more specific. Note that while the faster part 
of the relaxation is governed by binary solute-solvent interaction, the longtime decay is due to the collective fluctuation of the solvent cage, which must be coupled with the viscosity of the RTIL solvent.

\section{Supplementary Information (SI)}

Information regarding the radial distribution functions $(g(r))$ between $[\mathrm{Bmim}]\left[\mathrm{PF}_{6}\right]$ RTIL atoms in order to verify that the proper solvent equilibration, convergence of nonequilibrium and equilibrium solvation response functions, $g(r)$ between solute atoms and different carbon atoms of the side chain of the $[\mathrm{Bmim}]^{+}$cation, and the outline of the origin of nonpolar solvation dynamics of an excited solute in a solvent are available at www.ias.ac.in/chemsci.

\section{Acknowledgements}

SD acknowledges financial and computational support from Indian Institute of Technology, Patna, India. We acknowledge the anonymous reviewers for their sincere effort in improving the quality of the manuscript.

\section{References}

1. Welton T 1999 Room-Temperature Ionic Liquids. Solvents for Synthesis and Catalysis Chem. Rev. 992071

2. Hallett J P and Welton T 2011 Room-Temperature Ionic Liquids. Solvents for Synthesis and Catalysis 2 Chem. Rev. 1113508

3. MacFarlane D, Tachikawa N, Forsvth M, Pringle J M, Howlett P C, Elliott G D, Davis Jr. J H, Watanable M, Simon P and Angell C A 2014 Energy applications of ionic liquids Energy Environ. Sci. 7232

4. Bates E D, Mayton R D, Ntai I and Davis Jr. J H $2002 \mathrm{CO}_{2}$ Capture by a Task-Specific Ionic Liquid $J$. Am. Chem. Soc. 124926

5. Ramdin M, de Loos T W and Vlugt T J H 2012 Stateof-the-Art of $\mathrm{CO}_{2}$ Capture with Ionic Liquids Ind. Eng. Chem. Res. 518149

6. Mandal P K, Sarkar M and Samanta A 2004 ExcitationWavelength-Dependent Fluorescence Behavior of Some Dipolar Molecules in Room-Temperature Ionic Liquids J. Phys. Chem. A 1089048

7. Saha S, Mandal P K and Samanta A 2004 Solvation dynamics of Nile Red in a room temperature ionic liquid using streak camera Phys. Chem. Chem. Phys. 63106

8. Samanta A 2010 Solvation dynamics in ionic liquids: What we have learned from the dynamic fluorescence Stokes shift studies J. Phys. Chem. Lett. 11557

9. Samanta A 2006 Dynamic Stokes shift and excitation wavelength dependent fluorescence of dipolar molecules in room temperature ionic liquids J. Phys. Chem. B 110 13704

10. Arzhantsev S, Jin H, Baker G A and Maroncelli M 2007 Measurements of the Complete Solvation Response in Ionic Liquids J. Phys. Chem. B 1114978

11. Maroncelli M, Zhang X-X, Liang M, Roy D and Ernsting N P 2012 Measurements of the complete solvation response of coumarin 153 in ionic liquids and the accuracy of simple dielectric continuum predictions Faraday Discuss. 154409

12. Zhang X-X, Liang M, Ernsting N P and Maroncelli M 2013 Complete Solvation Response of Coumarin 153 in Ionic Liquids J. Phys. Chem. B 1174291

13. Zhang X-X, Liang M, Ernsting N P and Maroncelli M 2013 Conductivity and Solvation Dynamics in Ionic Liquids J. Phys. Chem. Lett. 41205

14. Zhang $X-X$, Liang $M$, Hunger $J$, Buchner $R$ and Maroncelli M 2013 Dielectric Relaxation and Solvation Dynamics in a Prototypical Ionic Liquid + Dipolar Protic Liquid Mixture: 1-Butyl-3-Methylimidazolium Tetrafluoroborate + Water J. Phys. Chem. B 11715356

15. Halder M, Headley L S, Mukherjee P, Song X and Petrich J W 2006 Experimental and Theoretical Investigations of Solvation Dynamics of Ionic Fluids: Appropriateness of Dielectric Theory and the Role of DC Conductivity $J$. Phys. Chem. A 1108623

16. Chakrabarty D, Chakraborty A, Seth D, Hazra P and Sarkar N 2004 Dynamics of solvation and rotational relaxation of Coumarin 153 in 1-butyl-3methylimidazolium hexafluorophosphate $[\mathrm{bmim}]\left[\mathrm{PF}_{6}\right]-$ water mixtures Chem. Phys. Lett. 397469

17. Chakrabarty D, Chakraborty A, Seth D and Sarkar N 2005 Effect of Water, Methanol, and Acetonitrile on Solvent Relaxation and Rotational Relaxation of Coumarin 153 in Neat 1-Hexyl-3-methylimidazolium Hexafluorophosphate J. Phys. Chem. A 1091764

18. Chakrabarty D, Seth D, Chakrabarty A and Sarkar N 2005 Dynamics of solvation and rotational relaxation of coumarin 153 in ionic liquid confined nanometer-sized microemulsions J. Phys. Chem. B 1095753

19. Adhikari A, Sahu K, Dey S, Ghosh S, Mandal U and Bhattacharyya K 2007 Femtosecond Solvation Dynamics in a Neat Ionic Liquid and Ionic Liquid Microemulsion: Excitation Wavelength Dependence J. Phys. Chem. B 11112809

20. Das S K, Sahu P K and Sarkar M 2013 DiffusionViscosity Decoupling in Solute Rotation and Solvent Relaxation of Coumarin153 in Ionic Liquids Containing Fluoroalkylphosphate (FAP) Anion: A Thermophysical and Photophysical Study J. Phys. Chem. B 117636

21. Das S K and Sarkar M 2012 Studies on the Solvation Dynamics of Coumarin 153 in 1-Ethyl-3Methylimidazolium Alkylsulfate Ionic Liquids: Dependence on Alkyl Chain Length ChemPhysChem 132761

22. Das S K, Sahu P K and Sarkar M 2013 Probing the Microscopic Aspects of 1-Butyl-3-Methylimidazolium Trifluoroacetate Ionic Liquid and Its Mixture with Water and Methanol: A Photophysical and Theoretical (DFT) Study J. Fluores. 231217

23. Horng M L, Gardecki J A, Papazyan A and Maroncelli M 1995 Subpicosecond Measurements of Polar Solvation Dynamics: Coumarin 153 Revisited J. Phys. Chem. 99 17311

24. Kashyap H K and Biswas R 2008 Dipolar Solvation Dynamics in Room Temperature Ionic Liquids: An Effective Medium Calculation Using Dielectric Relaxation Data J. Phys. Chem. B 11212431

25. Kashyap H K and Biswas R 2010 Solvation Dynamics of Dipolar Probes in Dipolar Room Temperature Ionic 
Liquids: Separation of Ion ${ }^{-}$Dipole and Dipole ${ }^{-}$Dipole Interaction Contributions J. Phys. Chem. B 114254

26. Kashyap H K and Biswas R 2010 Solvation Dynamics in Imidazolium and Phosphonium Ionic Liquids: Effects of Solute Motion Ind. J. Chem. 49A 685

27. Kashyap H K and Biswas R 2010 Stokes' Shift Dynamics in Imidazolium Ionic Liquids: Temperature Dependence J. Phys. Chem. B 11416811

28. Daschakraborty S and Biswas R 2011 Stokes' Shift Dynamics in Alkylimidazolium Aluminate Ionic Liquids: Domination of Solute-IL Dipole-Dipole Interaction Chem. Phys. Lett. $\mathbf{5 1 0} 202$

29. Daschakraborty S and Biswas R 2012 Stokes shift dynamics of [Na][TOTO] - a new class of ionic liquids: A comparative study with more common imidazolium analogs Chem. Phys. Lett. 54554

30. Daschakraborty S and Biswas R 2012 Ultrafast Solvation Response in Room Temperature Ionic Liquids: Possible Origin, and Importance of the Collective and the Nearest Neighbour Solvent Modes J. Chem. Phys. 137114501

31. Daschakraborty S, Pal T and Biswas R 2013 Stokes shift dynamics of ionic liquids: Solute probe dependence, and effects of self-motion, dielectric relaxation frequency window, and collective intermolecular solvent modes $J$. Chem. Phys. 139164503

32. Pal T and Biswas R 2014 Slow solvation in ionic liquids: Connections to non-Gaussian moves and multi-point correlations J. Chem. Phys. 141104501

33. Shim Y and Kim H J 2008 Dielectric Relaxation, Ion Conductivity, Solvent Rotation, and Solvation Dynamics in a Room-Temperature Ionic Liquid J. Phys. Chem. B 11211028

34. Shim Y and Kim H J 2013 Dielectric Relaxation and Solvation Dynamics in a Room-Temperature Ionic Liquid: Temperature Dependence. J. Phys. Chem. B 11711743

35. Terranova Z L and Corcelli S A 2013 On the Mechanism of Solvation Dynamics in Imidazolium-Based Ionic Liquids. J. Phys. Chem. B 11715659

36. Song X 2009 Solvation dynamics in ionic fluids: An extended Debye-Hückel dielectric continuum model $J$. Chem. Phys. 131044503

37. Kobrak M N 2007 A comparative study of solvation dynamics in room-temperature ionic liquids $\mathrm{J}$. Chem. Phys. 127184507

38. Shim Y, Duan J, Choi M Y and Kim H J 2003 Solvation in Molecular Ionic Liquids J. Chem. Phys. 1196411

39. Shim Y, Choi M Y and Kim H J 2005 A Molecular Dynamics Computer Simulation Study of RoomTemperature Ionic Liquids. II. Equilibrium and Nonequilibrium Solvation Dynamics J. Chem. Phys. 122044511

40. Kobrak M N 2006 Characterization of the Solvation Dynamics of an Ionic Liquid via Molecular Dynamics Simulation J. Chem. Phys. 125064502

41. Schmollngruber M, Schröder C and Steinhauser O 2013 Polarization Effects on the Solvation Dynamics of Coumarin C153 in Ionic Liquids: Components and Their Cross-Correlations J. Chem. Phys. 138204504

42. Wu E C and Kim H J 2016 MD Study of Stokes Shifts in Ionic Liquids: Temperature Dependence J. Phys. Chem. B 1204644

43. Muramatsu M, Nagasawa Y and Miyasaka Y 2011 Ultrafast Solvation Dynamics in Room Temperature Ionic
Liquids Observed by Three-Pulse Photon Echo Peak Shift Measurements J. Phys. Chem. A 1153886

44. Nagasawa Y and Miyasaka Y 2014 Ultrafast solvation dynamics and charge transfer reactions in room temperature ionic liquids Phys. Chem. Chem. Phys. 1613008

45. Ma J, Bout D V and Berg M 1995 Transient hole burning of $s$-tetrazine in propylene carbonate: A comparison of mechanical and dielectric theories of solvation J. Chem. Phys. 1039146

46. Fourkas J T, Benigno A and Berg M 1993 Time-resolved nonpolar solvation dynamics in supercooled and low viscosity $n$-butylbenzene J. Chem. Phys. 998552

47. Fourkas J T and Berg M 1993 Temperature-dependent ultrafast solvation dynamics in a completely nonpolar system J. Chem. Phys. 987773

48. Bagchi B 1994 Molecular theory of nonpolar solvation dynamics J. Chem. Phys. 1006658

49. Biswas R, Nandi N and Bagchi B 1997 Solvation dynamics in monohydroxy alcohols: Agreement between theory and different experiments J. Phys. Chem. B 101 2968

50. Reynolds L, Gardecki J A, Frankland S J V, Horng M L and Maroncelli M 1996 Dipole solvation in nondipolar solvents: experimental studies of reorganization energies and solvation dynamics J. Phys. Chem. 10010337

51. Saven J S and Skinner J L 1993 A molecular theory of the line shape: Inhomogeneous and homogeneous electronic spectra of dilute chromophores in nonpolar fluids J. Chem. Phys. 994391

52. Ladanyi B M and Stratt R M 1996 Short-Time Dynamics of Solvation: Relationship between Polar and Nonpolar Solvation J. Phys. Chem. 1001266

53. Ladanyi B M and Maroncelli M 1998 Mechanisms of solvation dynamics of polyatomic solutes in polar and nondipolar solvents: A simulation study J. Chem. Phys. 1093204

54. Pal T and Biswas R 2015 Composition dependence of dynamic heterogeneity time-and length scales in [Omim][BF4]/water binary mixtures: Molecular dynamics simulation study J. Phys. Chem. B 11915683

55. Carter E A and Hynes J T 1991 Solvation dynamics for an ion pair in a polar solvent: Time-dependent fluorescence and photochemical charge transfer J. Chem. Phys. 94 5961

56. Bernardi E, Martins M M and Stassen H 2005 The breakdown of linear response theory in non-polar solvation dynamics Chem. Phys. Lett. 407171

57. Faeder J and Ladanyi B M 2001 Solvation Dynamics in Aqueous Reverse Micelles: A Computer Simulation Study J. Phys. Chem. B $\mathbf{1 0 5} 11148$

58. Schmidt M W, Baldridge K K, Boatz J A, Elbert S T, Gordon M S, Jensen J H, Koseki S, Matsunaga N, Nguyen $\mathrm{K} \mathrm{A}, \mathrm{Su} \mathrm{S}$, Windus T L, Dupuis $\mathrm{M}$ and Montgomery Jr. J A 1993 General Atomic and Molecular Electronic Structure System J. Comput. Chem. 141347

59. Martínez L, Andrade R, Birgin E G and Martínez J M 2009 Packmol: A package for building initial configurations for molecular dynamics simulations J. Comput. Chem. 302157

60. Lopes J N C, Deschamps J and Pádua A A H 2004 Modeling Ionic Liquids Using a Systematic All-Atom Force Field J. Phys. Chem. B $\mathbf{1 0 8} 2038$ 
61. Jorgensen W L, Maxwell D S and Tirado-Rives J 1996 Development and Testing of the OPLS All-Atom Force Field on Conformational Energetics and Properties of Organic Liquids J. Am. Chem. Soc. 118 11225

62. Schroder C 1996 Comparing reduced partial charge models with polarizable simulations of ionic liquids Phys. Chem. Chem. Phys. 11811225

63. van der Spoel D, Lindahl E, Hess B, Groenhof G, Mark A E and Berendsen H J C GROMACS: Fast, Flexible, and Free J. Comput. Chem. 261701

64. Nosé S 1984 A unified formulation of the constant temperature molecular-dynamics methods J. Chem. Phys. 81 511

65. Hoover W G 1985 Canonical dynamics: Equilibrium phase-space distributions Phys. Rev. 311695

66. Berendsen H J C, Postma J P M, van Gunsteren W F, DiNola A and Haak J R 1984 Molecular-Dynamics with Coupling to an External Bath J. Chem. Phys. 81 3684

67. Darden T, York D and Pedersen L 1993 Particle mesh Ewald: An $N \cdot \log (N)$ method for Ewald sums in large systems J. Chem. Phys. 9810089

68. Maginn E J 2009 Molecular simulation of ionic liquids: current status and future opportunities J. Phys.: Condens. Matter 21373101

69. Pal T and Biswas R 2013 Rank-dependent orientational relaxation in an ionic liquid: an all-atom simulation study Theo. Chem. Acc. 1321348
70. Lopes J N C, Deschamps J and Pádua A A H 2006 Nanostructural organization in ionic liquids J. Phys. Chem. B 1103330

71. Morgado P, Shimizu K, José M S S, Esperança J M S S, Reis P M, Rebelo L P N, Lopes J N C and Filipe E J M 2013 Using ${ }^{129}$ Xe NMR to Probe the Structure of Ionic Liquids J. Phys. Chem. Lett. 42758

72. Hu Z and Margulis C J 2006 Heterogeneity in a roomtemperature ionic liquid: Persistent local environments and the red-edge effect Proc. Natl. Acad. Sci. U.S.A. 103 831

73. Richert R 2002 Heterogeneous liquids: fluctuations in space and time J. Phys. Condens. Matter. 14 R703

74. Jin H, Li X and Maroncelli M 2007 Heterogeneous Solute Dynamics in Room Temperature Ionic Liquids $J$. Phys. Chem. B 11113473

75. Wang Y and Voth G A 2005 Unique Spatial Heterogeneity in Ionic Liquids J. Am. Chem. Soc. 12712192

76. Triolo A, Russina O, Bleif H-J and Cola E D 2007 Nanoscale Segregation in Room Temperature Ionic Liquids J. Phys. Chem. B 1114641

77. Zheng Z-P, Gun W-H, Roy S, Mazur K, Nazet A, Buchner R, Bonn M and Hunger J 2015 Ionic Liquids: Not only Structurally but also Dynamically Heterogeneous Angew. Chem. Int. Ed. 54687

78. Roy D and Maroncelli M 2010 An improved four-site ionic liquid model. J. Phys. Chem. B 11412629

79. Egorov S A 2003 Nonpolar solvation dynamics in supercritical fluids J. Chem. Phys. 11810643 